\title{
Les grandes crues historiques de la Loire
}

\author{
M. Dacharry \\ Université des Sciences et Technologies de Lille
}

Le paradoxe du régime de la Loire est un lieu commun de la littérature ligérienne : d'une part un débit moyen relativement médiocre avec des basses eaux misérables, d'autre part des épisodes de crues exceptionnelles et ravageuses sur le long parcours du fleuve et sur ses affluents $[1,2,3]$. Si les basses eaux estivales, mal chronique de la Loire moyenne, et le risque de pénurie retiennent l'attention seulement depuis les années 50 , en revanche, les crues dans la mémoire des hommes sont liées à leurs installations les plus anciennes. Les premiers vestiges d'occupation humaine dans le Val se trouvent sur les micro-reliefs, monticules ou bourrelets alluviaux, insubmersibles [4], et les levées qui bordent presque continûment le lit mineur, sur plus de $500 \mathrm{~km}$ des plaines bourbonnaises jusqu'à Nantes, sont l'aboutissement des travaux collectifs millénaires par lesquels les riverains ont cherché à se mettre à l'abri des crues. Ce souci reste actuel. La hantise des conséquences funestes que pourraient avoir des événements comparables aux plus grandes crues du passé est sous-jacente à toutes les politiques d'aménagement du fleuve et de son bassin. Sur un pareil sujet, fort exploré par tant de spécialistes autorisés, il peut y avoir encore intérêt à réfléchir sur ce qu'on appelle les crues historiques, sur leur diversité et sur l'éventualité de leur retour.

\section{DEFINITION DE LA CRUE HISTORIQUE}

\subsection{Les crues exceptionnelles de la Loire moyenne}

Le terme d' «historique» est devenu habituel et quasi exclusif pour qualifier les trois débordements extraordinaires d'octobre 1846, de juin 1856, d'octobre 1866 dont l'ampleur n'a pas été dépassée depuis lors, entre le Bec d'Allier et la confluence de la Vienne. Ces événements aux conséquences désastreuses ont eu des précédents célèbres relatés dans des documents divers qui ont permis d'en établir la chronique, malheureusement lacunaire et imprécise à mesure qu'on remonte le cours des âges $[4,5]$. Ainsi sait-on avec certitude que des épisodes mémorables se sont produits aux dates suivantes :
- au temps où les turcies et levées n'étaient ni très hautes ni continues, $581,853,1231,1315,1346,1391,1426$, mai 1494 , mai $1519,1504,1522$, mai 1527 , mai 1549 , juin 1582 , septembre 1586 , octobre 1608 , novembre 1628 , janvier 1649 ;

- puis, malgré les renforcements de l'endiguement de la Loire aux XVII ème et XVIII ème siècles, octobre 1707, juin 1709 , novembre 1710 , février 1711 , mai 1733 , décembre 1755 , novembre 1790 .

\subsection{Critères de puissance des crues anciennes}

Les données hydrométriques qui permettent de caractériser et de comparer les grandes crues anciennes sont de trois espèces.

Significative est à cet égard l'étendue du champ d'inondation, en particulier la réunion en un seul plan d'eau du Loiret et de la Loire devant Orléans et celle du fleuve avec le Cher à Tours. En mai 1856, 100.000 hectares ont été recouverts par les eaux; pour cette raison, cette catastrophe est devenue crue de référence [ 6 ].

Le second indice trouve sa mesure dans l'ampleur des dommages causés aux hommes et aux biens : noyades, pertes de récoltes et de terres, destructions de ponts, ruptures de levées...» Dans l'esprit des populations, la grande crue extraordinaire est celle qui fait casser la levée» [ 4 ]. En mai 1856 , de Nevers à Nantes, près de 3.000 hectares de terres de culture ont été ravagés par ensablement ou érosion, à la suite de 160 brèches qui se sont produites dans les levées; les ponts de Fourchambault, Cosne, Sully ont été entièrement détruits, et ceux de St Thibault, Meung, Beaugency, Muides, Port Boulet, endommagés.

La hauteur aux échelles est l'information la plus précise et la plus instructive grâce aux archives des Ponts et Chaussées qui conservent des séries remarquables, surtout pour les années postérieures à la création en 1847 d'un service régulier d'annonce des crues, mais où se trouvent aussi des relevés antérieurs.

Le débit -des crues anciennes est estimé sur la base de l'hydrogramme de crue par la relation entre hauteurs et débits. Malheureusement les jaugeages récents ne peuvent 


\begin{tabular}{|l|c|c|c|}
\hline $\begin{array}{l}\text { Cotes sur la haute Loire } \\
\text { aux échelles de : }\end{array}$ & Goudet & Brives-Charensac & Bas-en-Basset \\
\hline 17 octobre 1846 & & $3,50 \mathrm{~m}$ & $7,10 \mathrm{~m}$ \\
29 mai 1856 & $12 \mathrm{~m}$ & & $6,25 \mathrm{~m}$ \\
26 septembre 1866 & & $6 \mathrm{~m}$ & $4,90 \mathrm{~m}$ \\
8 octobre 1878 & & $4,80 \mathrm{~m}$ & $6,50 \mathrm{~m}$ \\
$9-10$ octobre 1907 & & $4,80 \mathrm{~m}$ \\
$16-17$ octobre 1907 & $9 \mathrm{~m}$ & $6,10 \mathrm{~m}$ \\
22 octobre 1872 & & $6,38 \mathrm{~m}$ & $3,30 \mathrm{~m}$ \\
29 septembre 1890 & & & $4,40 \mathrm{~m}$ \\
4 novembre 1899 & & & $6,05 \mathrm{~m}$ \\
\hline
\end{tabular}

Tableau 1 : Hauteurs d'eau lors de différentes crues à Goudet, Brives-Charensac et Bas-en-Basset.

apporter qu'une faible garantie de contrôle à ces calculs. En effet, outre que les débits extraordinaires ne peuvent être jaugés, la largeur et la profondeur des lits sont rarement assez stables (surtout dans le Val) pour que les cotes relevées aujourd'hui aient à peu près la même signification qu'à une autre époque. Un calcul sommaire, fondé sur les traces certaines qu'ont laissées les plus fortes inondations, laisse penser que les eaux se sont (ou se seraient) élevées, si elles avaient pu se répandre librement dans le val, à un niveau de 5 mètres au-dessus de l'étiage. D'après la configuration morphologique, le débit correspondant pourrait atteindre entre 6000 et $9000 \mathrm{~m}^{3} / \mathrm{s}$ [4].

Après 150 ans de calculs et d'études, progressivement éclairés par des techniques renouvelées dans la mesure et la critique des données, il est admis que les débits maxima en $1846,1856,1866$, à peu près égaux après la confluence de la Loire et de l'Allier, ont été de l'ordre de $7600 \mathrm{~m}^{3} / \mathrm{s}$ à la confluence, de $7200 \mathrm{~m}^{3} / \mathrm{s}$ à Gien, de $5500 \mathrm{~m}^{3} / \mathrm{s}$ à Tours [6]. Les crues de la Loire sont, intrinséquement, les plus puissantes après la confluence de la Loire et de l'Allier parce qu'elles y sont l'addition des flux des deux artères maîtresses du haut bassin, presqu'égaux en volume et qui surviennent à peu près simultanément. Jusqu'à la confluence de la Vienne, l'absence d'affluents notables contribue à étaler les crues imparfaitement conjurées par les levées, et à écrêter l'onde progressant vers l'aval. La dernière évaluation du débit à $7600 \mathrm{~m}^{3} / \mathrm{s}$ au Bec d'Allier est sensiblement inférieure aux estimations antérieures des ingénieurs Sainjon ou Comoy $\left(8500\right.$ à $\left.9800 \mathrm{~m}^{3} / \mathrm{s}\right)$ et du Pr. Pardé $(8500$ à $\left.9000 \mathrm{~m}^{3} / \mathrm{s}\right)$, déjà légèrement revus à la baisse [1]. Finalement, ces différences entre les évaluations successives d'hydrologues expérimentés s'expliquent par les progrès acquis dans la connaissnce des variables à prendre en compte.

\subsection{Des crues mémorables ailleurs qu'en Loire moyenne}

Certes la crue de 1856 a été la plus haute, la plus longue, la plus désastreuse qui se soit jamais produite sur la Loire à partir de Roanne et sur l'Allier à partir de Vichy. Elle a rompu toutes les levées et inondé tous les vals endigués : sans les écoulements par les brèches, les niveaux aux échelles auraient été encore plus élevés. Cependant, fait notable, en amont du Bec d'Allier, les crues de 1846 et 1866 lui ont été supérieures. Sur la haute Loire, la crue de 1856 n'a rien eu d'exceptionnel et l'épisode «historique» est à d'autres dates (Tableau 1) :

La crue, violente et rapide, du 8 octobre I878, au caractère certainement «historique», $s$ 'est pour ainsi dire reproduite le 21 septembre 1980, faisant huit morts et un demimilliard de dégâts.

De même, à l'autre extrémité du cours, sur la basse Loire, après les confluences de la Vienne et de la Maine, les cotes relevées en 1856 ont été plusieurs fois dépassées. A Montjean, par exemple, la cote de $6,26 \mathrm{~m}$ (juin 1856) a été dépassée en février 1904, en décembre 1910, en avril 1919 , en mars 1923, en janvier 1936, en janvier 1941, en décembre 1952. Les débits ont été estimés à :

\begin{tabular}{|l|l|}
\hline $5.500 \mathrm{~m}^{3} / \mathrm{s}$ & en juin 1856 \\
$6.350 \mathrm{~m}^{3} / \mathrm{s}$ & en novembre-décembre 1910 \\
$6.160 \mathrm{~m}^{3} / \mathrm{s}$ & en janvier 1936 \\
$5.800 \mathrm{à} 5.900 \mathrm{~m}^{3} / \mathrm{s}$ & en février 1977 \\
$5.700 \mathrm{~m}^{3} / \mathrm{s}$ & en janvier 1982 \\
$6.500 \mathrm{~m}^{3} / \mathrm{s}$ & en décembre 1982 \\
$5.600 \mathrm{~m}^{3} / \mathrm{s}$ & en janvier 1994 \\
\hline
\end{tabular}

De fait, à la fin de sa course, le fleuve prend des caractères nouveaux sous l'influence de ses affluents tourangeaux et angevins, Cher, Indre, Vienne et Maine. Son débit moyen fait plus que doubler: $370 \mathrm{~m}^{3} / \mathrm{s}$ à Tours, $835 \mathrm{~m}^{3} / \mathrm{s}$ à Montjean en aval d'Angers. Mais, surtout, la répartition saisonnière des grandes crues y évolue, en gros, parallèlement à la courbe du régime mensuel moyen. Les plus grandes crues sont les plus fréquentes pendant la période des hautes eaux hivernales, contrairement à ce qui s'observe en Loire moyenne. A Gien, en effet, l'échantillon des très grandes crues, avec un maximum d'occurence pendant les trois mois de basses eaux, fait discordance à la fois dans le régime des débits moyens et dans la famille des crues. (Fig. 1.) 


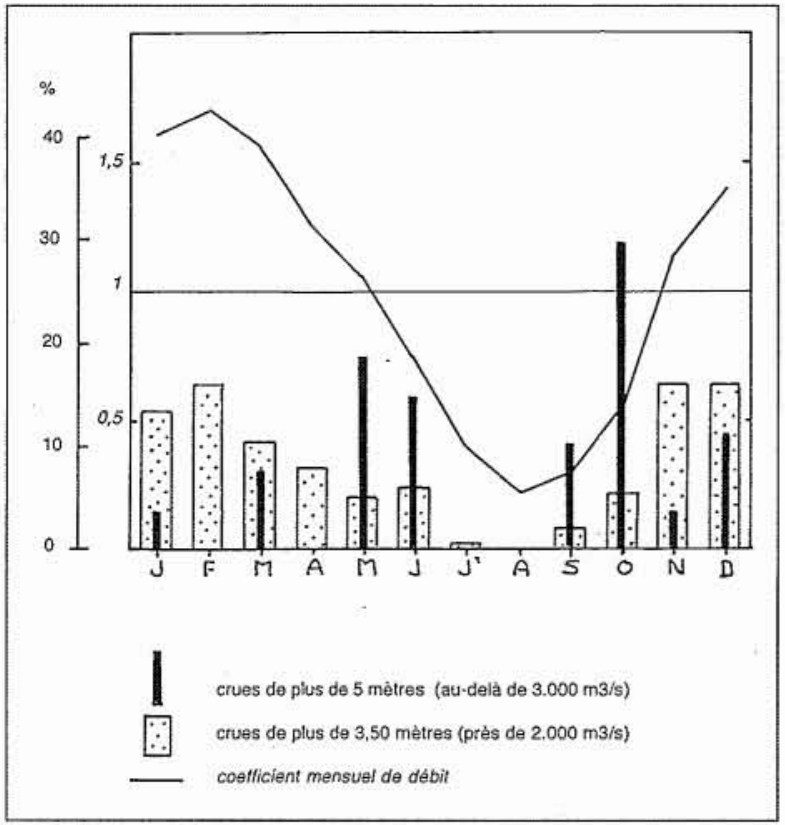

1. Histogrammes de fréquence des grandes crues de la Loire à Gien de 1825 à 1995 , comparés au régime moyen global

Enfin la liste serait longue des crues locales, rares et exceptionnelles, enregistrées sur les multiples artères du réseau de la Loire. Le 13 novembre 1790, l'Allier à I'échelle de Moulins est montée à $6,70 \mathrm{~m}$ (il était à $5,42 \mathrm{~m}$ en 1856). Sur le Cher, la crue de 1856 a été sensiblement moins forte que celles de 1608 et de 1743 [7]. Pour la Vienne, la crue mémorable ne se produit pas sous le Second Empire mais en février 1698 avec une cote de $6,78 \mathrm{~m}$ à Chatellerault et en juillet 1792, à Nouâtre, avec une cote record de $10,51 \mathrm{~m}$. De même, sur la Mayenne, les très grandes crues ont eu lieu en janvier 1881 et en novembre 1974 [8]

\section{II 圆 DIVERSITÉ DES CRUES ANCIENNES}

\subsection{Absence d'un modèle unique}

Il serait simpliste, pour des raisons de comparaison et de classement, de réduire les crues aux chiffres de débit et de hauteur à l'échelle. Ce peut être une forte tentation, notamment pour les crues anciennes, à mesure que le temps passe et que les circonstances perdent de leur relief et de leur clarté. Chaque événement, en vérité, est unique dans «... ce monde, extrêmement varié, d'événements et de causes, de types et d'exceptions ", pour reprendre l'expression de M. Pardé [1]. Sans aborder, ici, l'immense sujet de la genèse des crues de la Loire, traité ailleurs [2] et, ici, par D.Duband, et à s'en tenir au seul critère hydrométrique de la propagation du flot, les observations fort précises et déjà anciennes des ingénieurs du XIX ̀̀me siècle, notamment celles du fonds Paul Guillon, ont imposé la distinction fondamentale d'au moins huit grand types :

— crues limitées à la haute Loire seule (c'est-à-dire avant Roanne) ou très prépondérantes sur ce fleuve,

- crues touchant la haute Loire et le haut Allier (avant Parentignat-Vic-le Comte ),

- crues de la haute Loire et du haut Allier mais plus fortes sur l'Allier,

- crues de la haute Loire et du haut Allier s'étendant à tous les affluents du haut bassin,

- crues généralisées de la haute Loire et du haut Allier s'étendant au Cher, à l'Indre et à la Creuse,

- crues grossissant en descendant vers l'aval pour la Loire supérieure seule,

- crues grossissant en descendant vers l'aval, pour la Loire, l'Allier et légèrement pour le Cher,

- crues grossissant en descendant vers l'aval pour la Loire, l'Allier, le Cher, l'Indre et la Creuse.

De plus, pour affiner la distinction nécessaire au sein de chaque type de crues, il y aurait lieu, aussi, de se référer au volume net et non pas brut d'eau écoulée et de considérer la forme de l'hydrogramme. Ainsi les dernières estimations des débits maxima écoulés, au cours des trois grandes crues du XIXème siècle sur la Loire moyenne, sont les mêmes : 7 $600 \mathrm{~m}^{3} / \mathrm{s}$ au Bec d'Allier, $5500 \mathrm{~m}^{3} / \mathrm{s}$ à Blois [6] alors que la forme des hydrogrammes de 1846 et de 1866 , d'une part, et celle de 1856 , d'autre part, sont différentes. Les hydrogrammes du 23 octobre 1846 et du 29 septembre 1866, à Tours, se ressemblent mais le débit maximum de 1846 et le volume net sont sensiblement plus élevés que ceux de 1866 , de l'ordre, respectivement, de $10 \%$ et $30 \%$. Lorsqu'on considère ces deux crues, d'une part, et celle de 1856, crue d'été et non d'automne, d'autre part, les hydrogrammes n'ont plus rien de commun. On voit, sur celui de 1856 , d'abord deux intumescences (le 15 mai : $4,85 \mathrm{~m}$, le 21 mai : $5,28 \mathrm{~m}$ ) en précéder une troisième ( le 4 juin : $7,52 \mathrm{~m}$ ) qui fit céder les levées détrempées, foudroyante et catastro-

\begin{tabular}{|l|c|c|}
\hline & $\mathbf{7 ~ m a i ~ 1 9 4 0}$ & 26 janvier 1941 \\
\hline Débit maximum & $3026 \mathrm{~m}^{3} / \mathrm{s}$ & $3026 \mathrm{~m}^{3} / \mathrm{s}$ \\
Débit initial & $1160 \mathrm{~m}^{3} / \mathrm{s}$ & $280 \mathrm{~m}^{3} / \mathrm{s}$ \\
Volume brut & $2280 \mathrm{Mm}^{3}$ & $3525 \mathrm{Mm}^{3}$ \\
(total de l'intumescence) & $1340 \mathrm{Mm}^{3}$ & $3080 \mathrm{Mm}^{3}$ \\
Volume net & - & \\
\hline
\end{tabular}

Tableau 2. Comparaison des débits et volumes à Gien lors de deux crues. 


\begin{tabular}{|l|c|c|}
\hline & 6 novembre 1944 & 13 novembre 1958 \\
\hline Débit maximum & $1256 \mathrm{~m}^{3} / \mathrm{s}$ & $1250 \mathrm{~m}^{3} / \mathrm{s}$ \\
Débit initial & $528 \mathrm{~m}^{3} / \mathrm{s}$ & $185 \mathrm{~m}^{3} / \mathrm{s}$ \\
Volume brut & $550 \mathrm{Mm}^{3}$ & $1100 \mathrm{Mm}^{3}$ \\
(total de l'intumescence) & $232 \mathrm{Mm}^{3}$ & $710 \mathrm{Mm}^{3}$ \\
Volume net & & \\
\hline
\end{tabular}

Tableau 3. Comparaison des débits et volumes à Gien des crues de novembre 1944 et novembre 1958

phique, laquelle, en volume net, se révèle pourtant moindre que celle de 1846 , de $25 \%$ environ.

L'examen des différences pourrait être encore plus poussé en considérant la durée des submersions, la profondeur du champ d'inondation, la vitesse d'écoulement, la période de l'année, tous caractères de grande conséquence quant à l'annonce des crues, à l'organisation des secours et à l'ampleur des dommages.

La comparaison, à Gien (module : $335 \mathrm{~m}^{3} / \mathrm{s}$ ), d'épisodes de crues beaucoup plus modestes que les trois grandes crues du dix-neuvième siècle met en évidence la diversité cachée sous un même débit maximum (tableau 2). La crue de janvier 1941 est, en soi, bien plus remarquable que celle du 7 mai 1940.

En novembre 1944, en période de hautes eaux, des chutes de pluie médiocres ont suffi à gonfler les eaux du fleuve. En novembre 1958, quelques jours avant la montée des eaux, le débit moyen journalier était, seulement, de $185 \mathrm{~m}^{3} / \mathrm{s}$. Comparables tant par leur date que par leur élévation respective, les deux crues différent notablement par leur volume (tableau 3).

\subsection{Des concordances variées et imprévisibles}

L'examen attentif des fortes crues du passé, sur toutes les artères du bassin de la Loire, montre l'importance des phénomènes de concordance ou de discordance du maximum aux confluences des cours d'eau.

Certes la configuration immuable des diverses branches, petites et grandes, du réseau hydrographique dicte un certain degré de concordances et de combinaisons des flots élémentaires.

Par exemple, en cas de crue consécutive à une seule averse couvrant les hauts bassins de la Loire et de l'Allier, les phénomènes se développent comme en un mécanisme bien réglé. L'affluent, à cause de la pente de son talweg, de la forme de son lit, de la largeur de la vallée et du tracé de ses tributaires, est, au Bec d'Allier, en avance sur la Loire. Mais, à cause, aussi, des complications météorologiques et des décalages qui en résultent, l'avance naturelle de l'Allier sur la Loire (en 1846) est le plus souvent réduite (en 1866 et 1907), annulée (en 1872 et 1895), voire inversée (en 1790, 1856). Les inégalités en volume et les discordances des deux flots, dans le temps, sont irrégulières, comme sont très inégales, par leur intensité, leur évolution et leur extension, les pluies exceptionnelles sur tout le bassin avant cette confluence.

Aux autres confluences, la concordance ou le décalage des ondes sont également très variables au gré des averses successives ou simultanées qui gonflent les plus grands tributaires ligériens : le Cher qui draine un bassin aussi étendu que celui de l'Allier, la Vienne surtout $\left(21.000 \mathrm{~km}^{2}\right)$, la Maine (22 $185 \mathrm{~km}^{2}$ à Bouchemaine) dont les influences sont si décisives sur les plus fortes crues à Montjean [8]. Ici, en basse Loire, dans la chronique incomplète des crues historiques, il n'est pas d'exemple où les affluents d'aval auraient atteint tous à la fois des niveaux exceptionnels. Les maxima du fleuve, en aval de la Vienne, correspondent généralement à des flots non extraordinaires mais concordants des affluents. Par exemple, en décembre 1872, une seconde crue de la Vienne s'est cumulée, au confluent, avec une première crue de la Loire, d'où la hauteur exceptionnelle du fleuve à Saumur, Montjean et surtout à Nantes où elle est une des plus hautes connues.

Une combinaison du même genre s'est encore produite, sur la basse Loire, lors des grandes inondations de janvier 1843, de novembre-décembre 1910, de mars 1923 et, plus récemment, avec des variantes qui tiennent à l'infinie variété des averses locales, en février 1977, décembre 1982, janvier 1994. En janvier 1982, alors que le Cher, la Creuse, la Vienne et la Maine ont eu, chacun, des crues moyennes et que le maximum de la Vienne est passé quatre à cinq jours avant celui de la Loire, la hauteur du fleuve, aux Ponts -deCé, a été la même qu'en 1856 et 1910, et le débit à Montjean, a été légèrement supérieur à celui de 1910.

\section{1 LES GRANDES CATASTROPHES HISTORIQUES SONT-ELLES ENCORE POSSIBLES ?}

Deux catégories de causes conjointes peuvent conférer à une crue un caractère catastrophique : l'aléa hydrologique et la vulnérabilité des lieux.

\subsection{L'aléa physique et naturel}

Rien ne garantit que des événements hydrologiques comparables à ceux qui se sont déjà produits ne se reproduiront pas.

Même s'il y a des fluctuations climatiques qui font se 


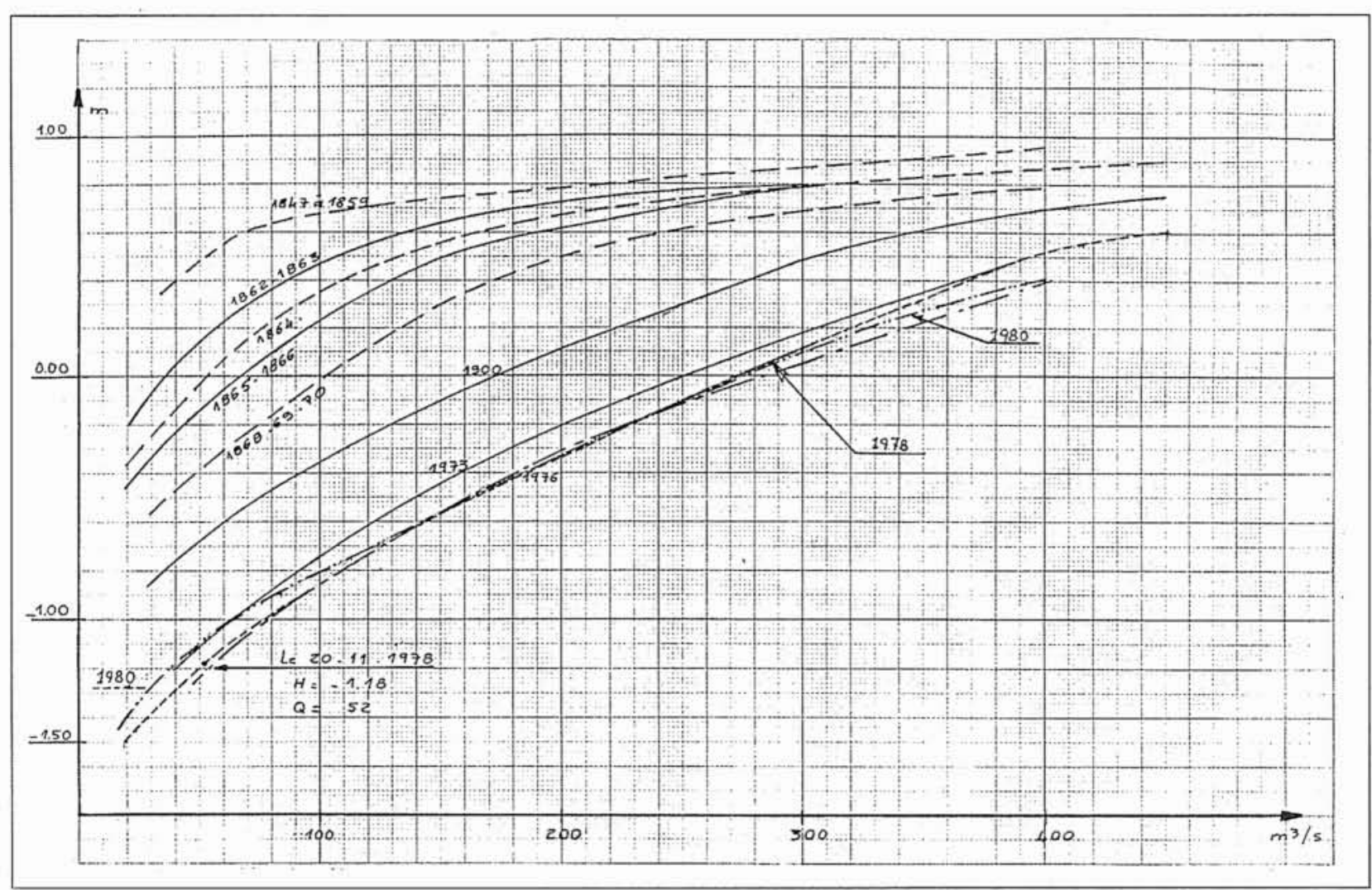

2. Courbes de correspondance hauteurs/débits à Orléans (Service hydrologique centralisateur du bassin Loire-Bretagne)

succéder - sans périodicité régulière d'ailleurs - séries d'années sèches et séries d'années humides [2,3], l'hydrologie de la Loire, avec des répartitions saisonnières toujours très changeantes d'une année à l'autre, ne s'est pas essentiellement modifiée. Des intensités pluviales récentes qui ont occasionné des crues «historiques» sur différentes artères du bassin de la Loire :

- en mai 1940, sur la Sioule et le Cher,

- en juin 1951, sur la Seuge,

- en octobre 1960, aux sources de la Vienne, du Cher et de la Creuse

- en septembre 1980, sur la haute Loire

en janvier 1982, sur la Loire entre Angers et Nantes,

- en décembre 1982 , sur le Clain $\left(310 \mathrm{~m}^{3} / \mathrm{s}\right.$ à Poitiers) et sur l'Indre $\left(500 \mathrm{~m}^{3} / \mathrm{s}\right.$ en aval de Loches),

ainsi que des averses répétées et prolongées sur une dizaine ou une vingtaine de jours, très comparables à celles de février 1977, janvier et décembre 1982, février 1988, janvier 1994, laissent penser que les mêmes causes peuvent produire les mêmes effets. Il n'y a aucune raison d'exclure l'éventualité de concordances désastreuses qui seraient le plus à craindre, sur la Loire moyenne, en mai-juin ou en automne. [2].

En revanche le régime de l'écoulement et, notamment, le risque d'inondation peuvent être modifiés par les changements du lit, naturels ou artificiels, et par les ouvrages régulateurs récents.
Les études minutieuses menées par le Service Hydrologique Centralisateur ont montré un fort abaissement général du fond du lit de la Loire du Bourbonnais à l'embouchure, depuis 1856, avec une accélération récente dans les années 1960-1970 [9]. L'abaissement n'est pas partout le même. Inexistant à l'endroit de seuils naturels ou artificiels (Briare, Amboise, St Mathurin) [10], il est plus fort au voisinage des grandes villes, comme à Orléans (1,50 m depuis 1856), à Tours (plus de $3 \mathrm{~m}$ ) (Fig.2). En certains secteurs, l'agrandissement consécutif de la section mouillée a été compensé par l'envahissement végétal du lit, du Bec d'Allier à Cosne et de St Mathurin à Montjean, par exemple. En cas de crue, par conséquent, il résulte de cet approfondissement général du lit un abaissement de la ligne d'eau pour un même volume écoulé. De fait, les levées ont résisté aux fortes crues de mars 1895, d'octobre 1907, de janvier 1924, de décembre 1944 et même, pour la plupart, à la crue de décembre 1982 dans le secteur de la Loire océanique. Elles doivent contenir, en principe, jusqu'à $6000 \mathrm{~m}^{3} / \mathrm{s}$.

L'effet du barrage écrêteur de crues de Villerest, mis en eau en 1985, ne doit pas non plus être négligeable. Pour les crues de période de retour supérieure à 50 ans, le débit maximum au Bec d'Allier pourrait être réduit de plusieurs centaines de $\mathrm{m}^{3} / \mathrm{s}$, et la ligne d'eau abaissée d'une quarantaine de centimètres. Les profondeurs de submersion en seraient réduites sans empêcher, toutefois, l'inondation totale du val à la fois par déversoir et par remous. 


\begin{tabular}{|l|l|}
\hline Q Max & Période de retour \\
\hline $3.000 \mathrm{~m}^{3} / \mathrm{s}$ & crue décennale \\
$6.000 \mathrm{~m}^{3} / \mathrm{s}$ & crue cinquantenale \\
$7.000 \mathrm{~m}^{3} / \mathrm{s}$ & crue centennale \\
$11.000 \mathrm{~m}^{3} / \mathrm{s}$ & crue millénale \\
\hline
\end{tabular}

Probabilité d'occurence des débits de la Loire au Bec d'Allier [6]

A supposer que les digues protectrices du lit mineur soient absolument résistantes [11], les crues extraordinaires seraient donc, aujourd'hui, moins menaçantes pour les riverains qu'il y a cent cinquante ans, en Loire moyenne et en Loire océanique.

\subsection{La vulnérabilité des lieux}

Cependant, transposer dans le présent les faits qui ont eu lieu dans le passé est une hypothèse difficile et toujours fragile car toutes les conditions qui tiennent à la configuration et à l'occupation des lieux ne sont jamais exactement les mêmes. L'espace urbanisé en zone inondable s'est considérablement étendu à la mesure du développement démographique et économique. Tours, par exemple, comptait, en 1856, 48000 habitants ; en 1990, l'agglomération en rassemblait 275000 .

Le montant des dommages directs que provoquerait, dans le Val de Loire, une crue telle que celle de 1856 a pu être estimé, en 1968 (pour une valeur des biens en 1962), à 381 millions de francs si les digues résistaient et à 1,3 millard si les digues étaient rompues [12]. En 1993, alors que 250.000 personnes vivent derrière les levées, J.P. Torterotot a estimé que les dommages d'une crue centennale $\left(7000 \mathrm{~m}^{3} / \mathrm{s}\right.$ au Bec d'Allier), écrêtée par le barrage de Villerest, seraient compris entre 3 et 10 milliards de francs selon les ruptures de déversoirs et de levées.

Cette protection des digues et levées, parade éternelle aux crues débordantes dans toutes les grandes vallées, l'est encore dans celle de la Loire où se vérifie particulièrement bien le schéma connu de l'augmentation des dommages à mesure que s'élève le niveau de protection [13] (Fig. 3). Les digues de la Loire sont périodiquement, après chaque désastre, consolidées, complétées, élargies, rehaussées (cf. prescriptions de Charlemagne et de Louis le Pieux en 819, ordonnances de Louis XI, de Henri II en 1551, de Charles IX en 1571, réglement de Colbert en 1668, et encore aux siècles suivants...) [4]. La grande crue de 1707 fit céder les levées en maints endroits et détermina un programme de nouveaux travaux, interrompus par les crues de $1709,1710,1711$ qui rouvrirent toutes les brèches. Nouveaux travaux, nouvelle crue de 1733, plus catastrophique que celle de 1707 : les levées sont rompues, les déversoirs défonçés. Le corsetage du lit fut renforcé pendant tout le XVIII ìme siècle; Nevers, Orléans, Blois, Tours, Saumur furent dotés de ponts de pierre plus solides etc. La grande crue de 1825, contenue dans les digues, inspira aux riverains une trompeuse confiance. Quand survin-
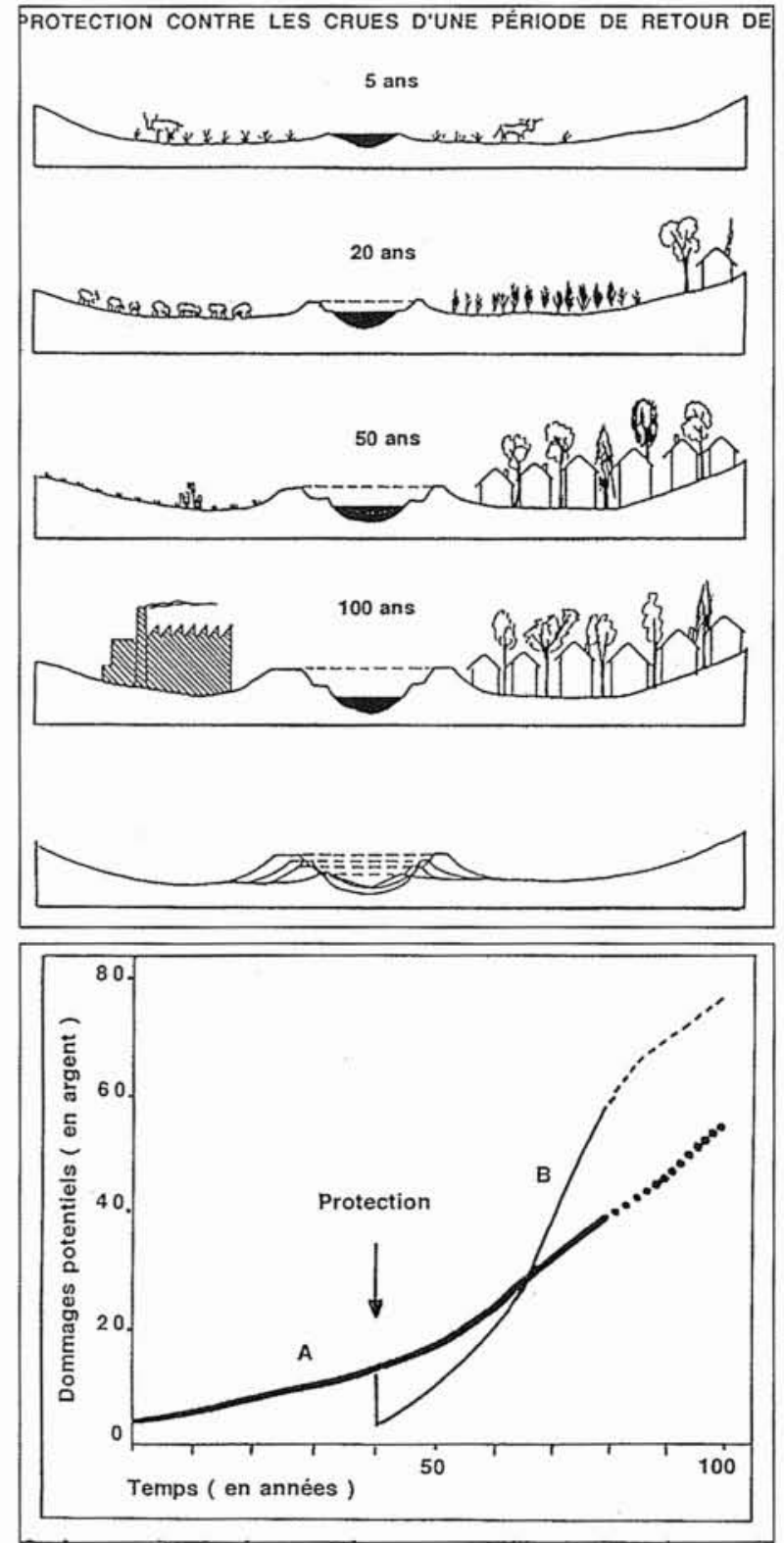

3. Augmentation des dommages à mesure que s'élève le niveau de protection (M. Dacharry, d'après ERICKSEN 1986) [13]

A. augmentation des pertes consécutives aux inondations dans le cas où aucun aménagement n'a contrarié l'écoulement naturel des eaux.

B. Evolution des pertes après l'édification d'ouvrages de protection.

rent les très grandes crues de 1846,1856, 1866, les désastres redoublèrent. A nouveau consolidées et entretenues, les levées ont résisté en mars 1895, octobre 1907, janvier I924, décembre 1944, jusqu'à nos jours...

Si la protection contre des crues relativement faibles et fréquentes a pour effet de valoriser des espaces, elle laisse les riverains sans défense contre les phénomènes rares mais beaucoup plus dommageables. 


\section{E CONCLUSION}

L'étude des crues historiques de la Loire ne devrait pas être interrompue parce que leur analyse peut encore être porteuse de leçons pour l'avenir. Entre le phénomène hydrologique qu'est l'inondation et le phénomène de risque, dû en grande partie à l'occupation inconsidérée des zones inondables par l'homme, il y a des compromis d'un équilibre fort délicat, plus qu'en tout autre grand bassin français sans doute, parce que les fantaisies naturelles y règnent. La configuration du réseau hydrographique de la Loire, son bassin étendu et disparate, très partagé entre des champs pluviaux mouvants et capricieux, la simultanéité possible d'averses d'origines différentes, peuvent créer des situations hydrologiques majeures imprévisibles.

\section{RÉFÉRENCES}

[1] PARDÉ M. «Etudes potamologiques sur la Loire et ses affluents», 183 p. NOROIS,Poitiers, $n^{\circ} 44$ bis, 1964.

PARDÉ M. «Sur la puissance des crues en diverses parties du monde», 293 p. GEOGRAPHICA, Zaragoza , 1961.

[2] DACHARRY M. «Hydrologie de la Loire en amont de Gien», 2 vol., 619 p., NEL, Paris, 1974.
[3] SCHULÉ C.A. «De Tours à Nantes, la Loire océanique, fleuve et flux», Cahiers Nantais, IGARUN, Nantes, $n^{\circ} 39-1993, p p$. 23-33.

[4] DION R. «Histoire des levées de la Loire», 312 p., Paris, 1961.

[5] GENESLAY E. «La Loire, crues et embâcles», 110 p., NEL. Paris, 1971.

[6] «ATLAS des zones inondables dans la vallée de la Loire», 17 volumes élaborés par la DIREN à partir de 1991, Service du Bassin Loire-Bretagne, Orléans La Source.

[7] GARNIER Ph. "Le Cher, étude hydrologique», 412 p. + annexes. Thèse de géographie, Université d'Angers, 1993.

[8] SCHULÉ C.A. «La Maine, contribution à la connaissance d'un hydrosystème», 1235 p. Thèse d'Etat, Nancy, 1984.

[9] ALLION Y., COULAUD D., CREPET F., FLEURY D., GAZOWSKI $\quad$ Z., GILLARDOT P. «La Loire», 189 p., Privat, Toulouse, 1994.

[10] BABONAUX Y.. «Le lit de la Loire», 252 p. Bibliothèque Nationale, Paris 1970.

[11] ARCHAMBAULT M., «Propositions pour la protection des Vals de Loire contre les crues dévastatrices du fleuve», NOROIS. Poitiers, $n^{\circ} 166$ - 1995, pp. $305-318$.

[12] FUNEL P., SCHWIRTZ M. «Etude économique de la protection des Vals de Loire par barrages écrêteurs de crues», S.H.F. Ximes Journées de l'Hydraulique, Paris, 1968.

[13] DACHARRY M. alnondations», Bulletin de l'Association de Géographes Français, 1990 - 1, pp.3-12. 